\title{
Reduction in the Number of Coupling Parameters
}

\author{
W. Zimmermann \\ Max-Planck-Institut für Physik und Astrophysik, Werner-Heisenberg-Institut für Physik, \\ D-8000 München 40, Federal Republic of Germany
}

Dedicated to the memory of Kurt Symanzik

\begin{abstract}
A method is developed for reducing the formulation of massless models with several independent couplings to a description in terms of a single coupling parameter. The original as well as the reduced system are supposed to be renormalizable and invariant under the renormalization group. For most models there are, if any, only a finite number of reductions possible including those which correspond to symmetries of the system. The reduction method leads to a consistent formulation of the reduced model in any order of perturbation theory even in cases where it is difficult to establish a symmetry in higher orders. An example where no symmetry seems to be involved is the interaction of a spinor field with a pseudoscalar field. For this model the reduction method determines the quartic coupling constant uniquely as a function of the Yukawa coupling constant. The Wess-Zumino model is an exceptional case for which the reduction method admits an infinite number of solutions besides the supersymmetric one.
\end{abstract}

\section{Introduction}

Symmetry considerations provide a natural method of reducing the number of independent parameters in models of quantum field theory. If a symmetry is imposed, otherwise unconstrained coupling parameters become related among each other so that the number of independent parameters is decreased. Renormalizability of the model is maintained provided anomalies are absent and the symmetry can be implemented in all orders of perturbation theory.

In this paper a more general approach for reducing the number of coupling parameters is taken which is based on the principles of renormalizability and invariance under the renormalization group. It turns out that these requirements severely limit the possibilities of constraining the coupling parameters to a single independent one. The method is developed for the reduction of massless models from $n+1$ coupling parameters $\lambda_{0}, \lambda_{1}, \ldots, \lambda_{n}$ to a description in terms of $\lambda_{0}$ only. Any symmetry requirement leading to a renormalizable formulation is certainly 
included by this treatment. In fact, hidden symmetries could be detected in this way. On the other hand there are cases where the general reduction is possible and unique, but no symmetry is known to be involved. It is also conceivable that a symmetry can only be implemented in low orders while the general reduction method leads to a unique prescription in all orders of perturbation theory. In such a case a renormalizable formulation of the reduced model is obtained for which, however, the relevant symmetry is only realized in low orders.

In Sect. 2 the general conditions are studied under which a reduction is possible. For the coupling parameters $\lambda_{j}$ as functions of $\lambda_{0}$ the ordinary differential equations

with

$$
\beta_{0} \frac{d \lambda_{j}}{d \lambda_{0}}=\beta_{j}
$$

$$
\lim _{\lambda_{0} \rightarrow 0} \lambda_{j}=0
$$

are found. $\beta_{j}$ denotes the $\beta$-function corresponding to $\lambda_{j}$. Equation (1.1) can be derived either from the Callan-Symanzik equations $[1,2]$ or the evolution equations of the effective couplings. An interesting possibility is the special case that the $\beta$-function of the reduced system vanishes identically ${ }^{1}$. Then, after inserting the functions $\lambda_{j}\left(\lambda_{0}\right)$ the $\beta$-functions of the original system also vanish identically

$$
\beta_{i} \equiv 0, \quad i=0,1, \ldots, n,
$$

and the system (1.1) is trivially satisfied.

Renormalizability for the original as well as the reduced system implies that the functions $\lambda_{j}\left(\lambda_{0}\right)$ allow for power series expansions in $\lambda_{0}$. In lowest order one finds a system of quadratic equations for the constant lowest order approximations $\varrho_{0}^{(j)}$ of the ratios

$$
\frac{\lambda_{j}}{\lambda_{0}}=\varrho_{0}^{(j)}+o\left(\lambda_{0}\right) .
$$

These are the eigenvalue conditions proposed by Chang for the ratios of coupling constants $[4]^{2}$. They form necessary conditions for the possibility of reducing the system. But without further restrictions they are not sufficient. For sometimes higher order effects prevent the extension of (1.4) to power series solutions of (1.1).

In Sect. 3 the case of two coupling parameters $g^{2}$ and $\lambda$ is treated in detail by applying results from $[6]$ and $[7]^{3}$. The $\beta$-functions are assumed to be of the form

$$
\beta_{0}=b_{0} g^{4}+\ldots, \quad \beta_{1}=c_{1} \lambda^{2}+c_{2} \lambda g^{2}+c_{3} g^{4}+\ldots .
$$

1 For some models arguments have been given indicating that the $\beta$-function vanishes to all orders of perturbation theory. See for instance [3]

2 Chang et al. applied the eigenvalue conditions to grand unification in order to build asymptotically free models with only one coupling constant. Unfortunately this program turned out to be too ambiguous due to the freedom in introducing heavy particles. See [5] which contains further references

3 The purpose of [6] was to find all asymptotically free solutions of the evolution equations with two coupling parameters. In this context the solutions of (1.1-2) were constructed by asymptotic expansions. Among the solutions found only the power series solutions are relevant for the present paper 
A reduction to a renormalizable description in terms of $g^{2}$ is only possible if the quadratic equation

$$
c_{1} \varrho_{0}^{2}+\left(c_{2}-b_{0}\right) \varrho_{0}+c_{3}=0
$$

has real roots, i.e. if the discriminant

$$
\Delta=\left(c_{2}-b_{0}\right)^{2}-4 c_{1} c_{3} \geqq 0
$$

is non-negative. For asymptotically free gauge theories with a Higgs coupling (1.7) coincides with the condition for asymptotic freedom found by Gross and Wilczek [8]. It is always satisfied for supersymmetric gauge theories where $\lambda=h^{2}$ with $h$ describing a matter or Higgs interaction. In lowest order the ratio of the coupling parameters is given by one of the roots $\varrho_{ \pm}$of $(1.6)$

Unless

$$
\frac{\lambda}{g^{2}}=\varrho_{ \pm}+o\left(g^{2}\right) \text {. }
$$

$$
\xi=-\frac{c_{1}}{b_{0}}\left(\varrho_{+}-\varrho_{-}\right) \quad\left(\varrho_{+} \geqq \varrho_{-}\right)
$$

is an integer the lowest order term (1.8) can be completed to a power series expansion in $g^{2}$. The precise conditions under which an expansion for integral $\xi$ is possible are stated in Sect. 4. It is further shown that by a reparametrization it can be arranged that the lowest order of a power series (1.8) becomes exact

and

$$
\lambda^{\prime}=\varrho_{ \pm} g^{2} \quad \text { if } \quad \varrho_{ \pm} \neq 0
$$

$$
\lambda^{\prime}=\varrho_{n} g^{2 n+2} \quad \text { if } \quad \lambda=o\left(g^{2 n+2}\right)
$$

with a suitably defined new coupling parameter $\lambda^{\prime}$.

In the remainder of the paper the reduction method is applied to two models of special interest: Sect. 4 concerns the interaction of a spinor field with a pseudoscalar field. For a consistent formulation of the renormalization it is necessary to introduce a quartic selfinteraction of a scalar field since the Yukawa interaction alone would not render the four pseudoscalar vertex part convergent. The model thus involves two independent coupling constants, $g$ for the Yukawa coupling and $\lambda$ for the quartic interaction. No symmetry is known which would relate the two coupling constants. While the bare scalar coupling constant cannot be dropped, one might think of setting the renormalized coupling constant $\lambda$ equal to zero in order to eliminate the additional parameter. However, formulations with different normalization points would then be inequivalent. On the other hand, the general reduction method leads to a unique power series expansion

$$
\lambda=\varrho_{ \pm} g^{2}+\varrho_{1} g^{4}+\ldots
$$

of $\lambda$, thus providing a consistent renormalizable description with one coupling constant $g$ only. The two values $\varrho_{+}$and $\varrho_{-}$correspond to different signs of $\lambda$.

Finally the reduction method is discussed for models which become supersymmetric by imposing relations among the coupling constants. Special problems may occur for models which are not asymptotically free. In Sect. 5 the massless Wess- 
Zumino model ${ }^{4}[9]$ is treated with independent coupling constants $g$ for the Yukawa and $\lambda$ for the quartic coupling. A peculiar situation is found due to the fact that $\xi$ is a negative integer

$$
\xi=-3 \text {. }
$$

This leads to an asymptotic expansion of $\lambda$ in the form ${ }^{5}$

$$
\lambda=\varrho_{+} g^{2}+\varrho_{1} g^{4}+\varrho_{2} g^{6}+\varrho_{3} g^{8}+d g^{8} \ln g^{2}+\ldots .
$$

The coefficient $\varrho_{3}$ is arbitrary and $d$ is determined uniquely by lower orders including the order $g^{6}$. For $d=0$ logarithms are absent and (1.14) represents a power series with arbitrary $\varrho_{3}$. Without using supersymmetry, calculations of order $g^{6}$ would be required to check whether or not $d=0$. But the existence of a renormalized supersymmetric formulation excludes the occurrence of logarithms so that $d=0$. With suitable supersymmetric normalization conditions one has

$$
\lambda=\varrho_{+} g^{2}
$$

for the supersymmetric solution and

$$
\lambda=\varrho_{+} g^{2}+\varrho_{3} g^{8}+\sum_{n=4}^{\infty} \varrho_{n} g^{2 n+2}
$$

with arbitrary $\varrho_{3}$. Thus the general reduction method is not unique in this special case, but also admits infinitely many asymmetric reduced systems ${ }^{6}$. Even the relation (1.15) is not characteristic for the supersymmetric case since by an asymmetric redefinition of $\lambda$ the relation (1.15) can always be restored.

No such problems seem to occur for supersymmetric models where the primary $\beta$-function is negative or vanishes in lowest order. For the $N=2$ and $N=4$ super Yang-Mills theories it was found that the relevant lowest order solutions can indeed be uniquely extended to power series expansions in the primary coupling constant [11]. General statements can be made about two-parametric models with $\beta$-functions of the form (1.5) and $b_{0} \leqq 0$. If $b_{0}=0$ and $\Delta>0$ two power series can be constructed for $\lambda$ with uniquely determined coefficients [7]. One of the expansions corresponds to the supersymmetric case. This includes a variety of models, in particular those which may have vanishing $\beta$-functions in any order of perturbation theory. If $b_{0}<0$ and $\Delta>0$ the model is asymptotically free. Usually supersymmetric models with asymptotic freedom are unstable against perturbations of the symmetry $[7,12]$. In the unstable case a unique power series for $\lambda$ can be constructed $[6,7]$. Thus in all these cases the general reduction method provides a unique formulation of the reduced model in every order of perturbation theory even though the symmetry may have been established for low orders only.

\footnotetext{
4 For the renormalization of the massless model see [10]

5 There is also a power series for $\lambda$ which is not related to supersymmetry

6 Recently it has been shown by O. Piguet and K. Sibold that there is only one realization of supersymmetry in the perturbative treatment of the massless Wess-Zumino model [17]. Therefore, the additional reduced systems do not seem to be supersymmetric
} 


\section{General Method of Reduction}

We consider a massless model of quantum field theory described by $n+1$ dimensionless coupling parameters $\lambda_{0}, \lambda_{1}, \ldots, \lambda_{n}$ and a normalization mass $\kappa$. The model is supposed to be invariant under the renormalization group. Our aim is to express $\lambda_{1}, \ldots, \lambda_{n}$ as functions of $\lambda_{0}$ so that a model involving a single coupling parameter $\lambda_{0}$ is obtained which is again invariant under the renormalization group. Accordingly we write each $\lambda_{j}$ as a function of

$$
\lambda_{j}=\lambda_{j}\left(\lambda_{0}\right),
$$

independent of the normalization mass $\kappa$. The functions $\lambda_{j}\left(\lambda_{0}\right)$ should be differentiable in the domain of $\lambda_{0}$ considered and vanish in the weak coupling limit $^{7}$

$$
\lim _{\lambda_{0} \rightarrow 0} \lambda_{j}\left(\lambda_{0}\right)=0 .
$$

For the Green's functions of the original system the invariance under the renormalization group implies the Callan-Symanzik equations

$$
\left(\kappa^{2} \frac{\partial}{\partial \kappa^{2}}+\sum \beta_{j} \frac{\partial}{\partial \lambda_{j}}+\gamma\right) \tau=0,
$$

while for the Green's functions of the reduced system the equations

$$
\left(\kappa^{2} \frac{\partial}{\partial \kappa^{2}}+\beta^{\prime} \frac{\partial}{\partial \lambda_{0}}+\gamma^{\prime}\right) \tau^{\prime}=0
$$

follow. The $\beta$ - and $\gamma$-functions depend on the coupling parameters only. $\beta^{\prime}$ and $\gamma^{\prime}$ are functions of the single variable $\lambda_{0} . \gamma$ and $\gamma^{\prime}$ are additive in the contributions from the field operators occurring in the Green's functions. $\tau$ is a function of the momenta, the coupling parameters and the normalization mass $\kappa . \tau^{\prime}$ is obtained from $\tau$ by substituting the functions (2.1) for the parameters $\lambda_{j}$. Accordingly,

$$
\frac{\partial \tau^{\prime}}{\partial \lambda_{0}}=\frac{\partial \tau}{\partial \lambda_{0}}+\sum_{j=1}^{n} \frac{\partial \tau}{\partial \lambda_{j}} \frac{d \lambda_{j}}{d \lambda_{0}} .
$$

Linear independence of the Green's functions and their derivatives leads to the relations

$$
\beta^{\prime}=\beta_{0}, \gamma^{\prime}=\gamma, \beta^{\prime} \frac{d \lambda_{j}}{d \lambda_{0}}=\beta_{j} .
$$

Hence the functions (2.1) must satisfy the following system of ordinary differential equations

$$
\beta_{0} \frac{d \lambda_{j}}{d \lambda_{0}}=\beta_{j} .
$$

On the other hand, if the functions (2.1) satisfy (2.5), the reduced form (2.4) of the Callan-Symanzik equations follows. Thus the system (2.5) forms a necessary and sufficient condition for reducing the original system by the functions $\lambda_{j}\left(\lambda_{0}\right)$.

7 The condition of renormalizability requiring that the functions $\lambda_{j}$ can be expanded with respect to powers of $\lambda_{0}$ will not be used for the time being 
It is instructive to use an alternative method for the derivation of (2.5) by eliminating the scale variable from the evolution equations of the effective couplings. At a normalization mass $\kappa^{\prime 2}$ we impose on the coupling parameters that the values $\lambda_{j}^{\prime}$ of $\lambda_{j}$ are given functions of the value $\lambda_{0}^{\prime}$ of $\lambda_{0}$,

$$
\lambda_{j}^{\prime}=\lambda_{j}\left(\lambda_{0}^{\prime}\right) \text { at } \kappa^{2}=\kappa^{\prime 2} \text {. }
$$

We want to investigate under which restrictions on the functions the same dependence holds at other normalization points:

$$
\lambda_{j}=\lambda_{j}\left(\lambda_{0}\right) \text { at } \kappa^{2} .
$$

If the normalization mass is changed from $\kappa^{\prime 2}$ to $\kappa^{2}$ the field operators $\varphi_{i}$ of the system undergo a transformation of the renormalization group,

$$
\varphi_{j}\left(x, \lambda_{0}, \ldots, \lambda_{n}, \kappa^{2}\right)=z_{j}^{1 / 2} \varphi_{j}\left(x, \lambda_{0}^{\prime}, \ldots, \lambda_{n}^{\prime}, \kappa^{\prime 2}\right)
$$

with positive $z_{j}{ }^{8}$. The new values of the coupling parameters are given by

$$
\begin{aligned}
\lambda_{0} & =\bar{\lambda}_{0}\left(u, \lambda_{0}^{\prime}, \lambda_{1}^{\prime}, \ldots, \lambda_{n}^{\prime}\right), \\
\lambda_{j} & =\bar{\lambda}_{j}\left(u, \lambda_{0}^{\prime}, \lambda_{1}^{\prime}, \ldots, \lambda_{n}^{\prime}\right), \\
u & =\kappa^{2} / \kappa^{\prime 2}, \quad j=1, \ldots, n .
\end{aligned}
$$

The functions $\bar{\lambda}_{0}, \bar{\lambda}_{j}$ denote effective couplings suitably defined as analytic functions of $u$ which are regular at any positive value of $u^{9}$.

In order to determine the constraints on the functions $\lambda_{j}$ we take a fixed initial value $\lambda_{0}^{\prime} \neq 0$, and first discuss the case where

$$
\beta_{0}\left(\lambda_{0}^{\prime}, \lambda_{1}^{\prime}, \ldots, \lambda_{n}^{\prime}\right) \neq 0 \text {. }
$$

Expression (2.10) equals the value of $\partial \bar{\lambda}_{0} / \partial u$ at $u=1, \lambda_{0}=\lambda_{0}^{\prime}, \lambda_{j}=\lambda_{j}^{\prime}$. Since $\bar{\lambda}_{0}$ is regular analytic at $u=1$ the derivative $\partial \bar{\lambda}_{0} / \partial u$ is continuous near $u=1$ so that

$$
\frac{\partial \bar{\lambda}_{0}}{\partial u}\left(u, \lambda_{0}^{\prime}, \lambda_{1}^{\prime}, \ldots, \lambda_{n}^{\prime}\right) \neq 0
$$

in a neighborhood of $u=1$. Therefore, Eq. (2.8) can be inverted with respect to $u$. Inserting the inversion

$$
u=u\left(\lambda_{0} ; \lambda_{0}^{\prime}, \lambda_{1}^{\prime}, \ldots, \lambda_{n}^{\prime}\right)
$$

into (2.9) we find that the $\lambda_{j}$ necessarily become functions of $\lambda_{0}$ which are independent of the normalization mass $\kappa$. By definition they represent the functions $\lambda_{j}$ in $(2.6-7)$ :

$$
\lambda_{j}\left(\lambda_{0}\right)=\bar{\lambda}_{j}\left(u\left(\lambda_{0} ; \lambda_{0}^{\prime}, \ldots, \lambda_{n}^{\prime}\right), \lambda_{0}^{\prime}, \ldots, \lambda_{n}^{\prime}\right) .
$$

With the help of the evolution equations

$$
u \frac{\partial \bar{\lambda}_{i}}{\partial u}=\beta_{i}\left(\bar{\lambda}_{0}, \lambda_{1}\left(\bar{\lambda}_{0}\right), \ldots, \lambda_{n}\left(\bar{\lambda}_{0}\right)\right), \quad i=0,1, \ldots, n
$$

8 For the concept of the renormalization group used here see [13]

9 For the possibility of defining effective couplings as analytic functions see [14] 
the scale variable $u$ can be eliminated near $u=1$. We thus obtain (2.5) in the form

$$
\frac{d \lambda_{j}}{d \lambda_{0}}=\frac{\beta_{j}\left(\lambda_{0}, \lambda_{1}, \ldots, \lambda_{n}\right)}{\beta_{0}\left(\lambda_{0}, \lambda_{1}, \ldots, \lambda_{n}\right)}, \quad j=1, \ldots, n,
$$

valid in the neighborhood of $\lambda_{0}=\lambda_{0}^{\prime}$.

We next discuss the case where

$$
\beta_{0}\left(\lambda_{0}^{\prime}, \lambda_{1}^{\prime}, \ldots, \lambda_{n}^{\prime}\right)=0
$$

In this case the function $\beta_{0}\left(\lambda_{0}, \lambda_{1}\left(\lambda_{0}\right), \ldots, \lambda_{n}\left(\lambda_{0}\right)\right)$ has a zero at $\lambda_{0}=\lambda_{0}^{\prime}$. Then $(2.13)$ implies

for the function

$$
\frac{\partial \bar{\lambda}_{0}}{\partial u}=0 \quad \text { at } \quad u=1
$$

$$
\bar{\lambda}_{0}=\bar{\lambda}_{0}\left(u, \lambda_{0}^{\prime}, \lambda_{1}^{\prime}, \ldots, \lambda_{n}^{\prime}\right)
$$

Since $\bar{\lambda}_{0}$ is regular analytic in $u$ at $u=1$ it is

or

$$
\frac{\partial \bar{\lambda}_{0}}{\partial u} \neq 0 \text { for } u \neq 1 \text { near } u=1
$$

$$
\frac{\partial \bar{\lambda}_{0}}{\partial u} \equiv 0
$$

Hence the function (2.16) is either variable in $u$ and stationary at $u=1$ or it is constant ${ }^{10}$.

In case (2.17) of variable $\bar{\lambda}_{0}$ we may invert (2.8) for $u<1$ as well as $u>1$, obtaining

$$
\begin{aligned}
& u=u_{-}\left(\lambda, \lambda_{0}^{\prime}, \lambda_{1}^{\prime}, \ldots, \lambda_{n}^{\prime}\right) \text { for } u<1, \\
& u=u_{+}\left(\lambda, \lambda_{0}^{\prime}, \lambda_{1}^{\prime}, \ldots, \lambda_{n}^{\prime}\right) \text { for } u>1 .
\end{aligned}
$$

If $\bar{\lambda}_{0}$ has an extremal value at $u=1$ the inversions $u_{-}$and $u_{+}$denote different branches of $u$ both defined for $\lambda_{0}<\lambda_{0}^{\prime}$ in case of a maximum or $\lambda_{0}>\lambda_{0}^{\prime}$ in case of a minimum. Inserting (2.19) and (2.20) into (2.9) we find two sets of functions $\lambda_{j}$ of $\lambda_{0}$ which must be identical to (2.6) and thus to each other. For $\lambda_{0} \neq \lambda_{0}^{\prime}$ again (2.5) follows. Equation (2.5) can be extended to $\lambda_{0}=\lambda_{0}^{\prime}$ by taking the limit $\lambda_{0} \rightarrow \lambda_{0}^{\prime}$.

We now turn to the case (2.18) of constant $\bar{\lambda}_{0}$. Equations (2.7) and (2.8) imply that $\lambda_{j}$ does not depend on $u$ either, so that by (2.13) also the other $\beta$-functions vanish. The system (2.5) is then trivially satisfied.

We summarize the results as follows: In all cases the functions satisfy the system (2.5) of ordinary differential equations in agreement with the derivation given in the first part of this section. If $\lambda_{0}$ is a zero of one of the $\beta$-functions - with the other coupling parameters expressed as functions (2.6) of $\lambda_{0}^{\prime}-$ the system (2.5) implies that $\lambda_{0}^{\prime}$ is a zero of all $\beta$-functions. It follows that the effective couplings are either variable in $u$ and stationary at $u=1$ or they are all independent of $u$.

We provide some further information on the zeroes of the $\beta$-functions considered. Zeroes of the first type with variable effective couplings are always

10 For a discussion of zeroes of the $\beta$-function which correspond to stationary values of the effective coupling see [14] 
isolated. For it is

$$
\beta\left(\bar{\lambda}_{0}, \lambda_{1}\left(\bar{\lambda}_{0}\right), \ldots, \lambda_{n}\left(\bar{\lambda}_{0}\right)\right)=\frac{\partial \bar{\lambda}_{0}}{\partial u} \neq 0 \text { for } \quad \bar{\lambda}_{0} \neq \lambda_{0}^{\prime}
$$

in a neighborhood of $\lambda_{0}^{\prime}$. We further observe that a zero must necessarily be of the second type with constant effective couplings if all derivatives

$$
\frac{d^{m}}{d \lambda_{0}^{m}} \beta\left(\lambda_{0}, \lambda_{1}\left(\lambda_{0}\right), \ldots, \lambda_{n}\left(\lambda_{0}\right)\right)
$$

exist at $\lambda_{0}=\lambda_{0}^{\prime}$. For then all derivatives of $\bar{\lambda}_{0}$ with respect to $u$ vanish at $u=1$ as follows by differentiating the evolution equation (2.13) of $\bar{\lambda}_{0}$. Sufficient for the existence of the derivatives (2.21) is the existence of all partial derivatives of $\beta^{11}$. The functions $\lambda_{j}\left(\lambda_{0}\right)$ were assumed to be differentiable and the existence of their higher derivatives follows by differentiating the system $(2.5)$.

Zeroes of the $\beta$-functions with the effective couplings independent of the scale variable need not be isolated. In fact, arguments have been given for some supersymmetric models that reduced forms exist with $\beta$-functions vanishing in any order of perturbation theory. If this should prevail independent of perturbation theory the relations

$$
\beta_{i}\left(\lambda_{0}, \lambda_{1}\left(\lambda_{0}\right), \ldots, \lambda_{n}\left(\lambda_{0}\right)\right)=0, \quad i=0,1, \ldots, n,
$$

would vanish identically in $\lambda_{0}$ for some functions $\lambda_{j}\left(\lambda_{0}\right)$.

The reducibility condition (2.5) allows for a large class of solutions unless further restrictions are imposed. In a region of non-vanishing $\beta_{0}$ the Lipschitz condition can be verified for the ratios $\beta_{j} / \beta_{0}$ provided certain differentiability assumptions on the $\beta$-functions are made. With this the Picard-Lindelöf theorem applies according to which exactly one solution $\lambda_{j}\left(\lambda_{0}\right)$ of $(2.5)$ passes through any point $\lambda_{0}^{\prime}, \lambda_{1}^{\prime}, \ldots, \lambda_{n}^{\prime}$. Due to the singular nature of the system (2.5) at $\lambda_{0}=\lambda_{j}=0$, the standard existence theorems cannot be applied there. On the other hand it is difficult to gain control over the asymptotic behavior in the weak coupling limit for solutions with prescribed non-vanishing initial values $\lambda_{0}^{\prime}, \ldots, \lambda_{n}^{\prime}$. In general, uniqueness properties do not hold for solutions passing through the origin $\lambda_{0}=\lambda_{j}=0$ : For some systems there are no solutions of (2.5) which satisfy (2.2). For others there are infinitely many such solutions.

Further constraints are imposed if we require renormalizability for the original as well as the reduced system. Then the Green's functions of the original system have power series expansions in $\lambda_{0}, \lambda_{1}, \ldots, \lambda_{n}$ and the Green's functions of the reduced system can be expanded with respect to powers of $\lambda_{0}{ }^{12}$. This leads to the requirement that the solutions $\lambda_{j}\left(\lambda_{0}\right)$ of $(2.5)$ possess power series expansions in $\lambda_{0}$.

It is easy to work out the conditions necessary for the renormalizable reduction of a system in lowest order of the primary coupling constant. As example we

11 A stationary value of the effective coupling indeed leads to a singular behavior for the derivatives of the $\beta$-function (see [14])

12 We do not consider here expansions with respect to fractional powers or logarithms of coupling constants which may arise due to infrared singularities of conventional perturbation theories (see for instance $[15,16]$ ) 
consider $\beta$-functions with the expansions

$$
\begin{aligned}
\beta_{0}= & b_{0} \lambda_{0}^{2}+\sum_{n=3}^{\infty} \sum_{m=0}^{n-1} \sum_{j_{1} \ldots j_{m}} b_{n-m, j_{1} \ldots j_{m}} \lambda_{0}^{n-m} \lambda_{j_{1}} \ldots \lambda_{j_{m}}, \\
\beta_{j}= & \sum_{i k} c_{i k}^{(j)} \lambda_{i} \lambda_{k}+\sum_{i} c_{i}^{(j)} \lambda_{i} \lambda_{0}+c^{(j)} \lambda_{0}^{2} \\
& +\sum_{n=3}^{\infty} \sum_{m=0}^{n} \sum_{j_{1} \ldots j_{m}} c_{n-m, j_{1} \ldots j_{m}} \lambda_{0}^{n-m} \lambda_{j_{1}} \ldots \lambda_{j_{m}}
\end{aligned}
$$

where $\lambda_{0}$ is the square $\lambda_{0}=g^{2}$ of the primary coupling parameter $g$. Since all $\beta$-functions are even functions of $g$ it is natural to require that the coupling parameters $\lambda_{j}$ of the reduced system are also even in $g$. Renormalizability combined with the condition (2.2) implies that the coupling parameters of the reduced system have power series expansions

$$
\lambda_{j}=\varrho_{0}^{(j)} g^{2}+\sum_{n=2}^{\infty} \varrho_{n}^{(j)} g^{2 n+2} .
$$

Comparing the coefficients of $g^{4}$ in (2.5) we find the quadratic equations [4]

$$
\sum_{i k} c_{i k}^{(j)} \varrho_{0}^{(i)} \varrho_{0}^{(k)}+\sum_{i}\left(c_{i}^{(j)}-\delta_{i j} b_{0}\right) \varrho_{0}^{(i)}+c^{(j)}=0 .
$$

Its solutions $\varrho_{0}^{(j)}$ represent the lowest order values of the ratios $\lambda_{j} / g^{2}$. As such they should be real and - if required by the model - satisfy constraints like the positivity of coupling parameters. The equations (2.26) are necessary for the renormalizability of the reduced system, but not always sufficient. For in some cases the lowest order approximation based on a solution of (2.26) cannot be extended to power series expansions. Examples for that will be found in the following section.

\section{Two Coupling Parameters}

We are going to discuss in some detail the reduction of systems involving two coupling constants. The notation used is

$$
\left(\kappa^{2} \frac{\partial}{\partial \kappa^{2}}+\beta_{0}\left(g^{2}, \lambda\right) \frac{\partial}{\partial g^{2}}+\beta_{1}\left(g^{2}, \lambda\right) \frac{\partial}{\partial \lambda}+\gamma\right) \tau=0
$$

for the Callan-Symanzik equations. The $\beta$-functions are assumed to have expansions of the form

$$
\begin{aligned}
\beta_{0}= & b_{0} g^{4}+\sum_{n=3}^{\infty} \sum_{m=0}^{n-1} b_{n-m, m} g^{2(n-m)} \lambda^{m}, \\
\beta_{1}= & c_{1} \lambda^{2}+c_{2} \lambda g^{2}+c_{3} g^{4} \\
& +\sum_{n=3}^{\infty} \sum_{m=0}^{n} c_{n-m, m} g^{2(n-m)} \lambda^{m},
\end{aligned}
$$


which cover a large variety of models. We want to investigate under which conditions the model can be reduced by

$$
\lambda=\lambda\left(g^{2}\right)
$$

to a renormalizable system involving a single coupling constant $g$. The reducibility condition (2.5) takes the form

$$
\beta_{0} \frac{d \lambda}{d g^{2}}=\beta_{1}
$$

Renormalizability and condition (2.2) impose on the solutions that they can be expanded in the form

$$
\lambda=\varrho_{0} g^{2}+\sum_{j=1}^{\infty} \varrho_{j} g^{2 j+2}
$$

The first coefficient $\varrho_{0}$ is determined to be a root of the quadratic equation

$$
c_{1} \varrho_{0}^{2}+\left(c_{2}-b_{0}\right) \varrho_{0}+c_{3}=0 .
$$

$\varrho_{0}$ is only real if the discriminant

is non-negative

$$
\Delta=\left(c_{2}-b_{0}\right)^{2}-4 c_{1} c_{2}
$$

$$
\Delta \geqq 0
$$

This requirement already precludes the reduction for a large number of models. In the work that follows (3.9) will be assumed. There may be further restrictions on the values of the first coefficients $\varrho_{0}$. For instance, in some models $\lambda$ is the square of a coupling parameter and cannot be negative for that reason. In this case only nonnegative values of $\varrho_{ \pm}$are admissible.

For the case

$$
b_{0} \neq 0, \quad c_{1} \neq 0,
$$

we may take over the results obtained in [6] concerning power series solutions of (3.5). The following notations will be used. $\varrho_{ \pm}$denotes the roots of (3.7) with $\varrho_{+}$ being the larger value,

A number $\xi$ is defined by

$$
\varrho_{+} \geqq \varrho_{-} .
$$

$$
\xi=-\frac{c_{1}}{b_{0}}\left(\varrho_{+}-\varrho_{-}\right), \quad\left(b_{0} \neq 0\right)
$$

Since usually $c_{1}>0$ positive $\xi$ implies asymptotic freedom.

If $\xi<0$ a power series solution

$$
\lambda_{-}=\varrho_{-} g^{2}+\sum_{j=1}^{\infty} \varrho_{-j} g^{2 j+2}
$$

of (3.5) exists with uniquely determined coefficients. Further the solution

$$
\lambda_{+}=\varrho_{+} g^{2}+\sum_{j=1}^{\infty} \varrho_{+j} g^{2 j+2}
$$


exists if $\xi<0$ is not integral. If $\xi$ is a negative integer one finds the general solution of (3.5) as an expansion involving logarithms

$$
\lambda_{+}=\varrho_{+} g^{2}+\sum_{j=1}^{|\xi|-1} \varrho_{+j} g^{2 j+2}+\varrho_{+|\xi|} g^{2 \xi+2}+d g^{2|\xi|+2} \ln g^{2}+\ldots
$$

The coefficient $\varrho_{+|\xi|}$ is arbitrary, the coefficient $d$ of the first logarithmic term is uniquely determined by lower orders. If $d=0$ no power series solution $\lambda_{+}$of (3.5) exists. In that case a solution with asymptotic behavior $\varrho_{+} g^{2}$ for $g \rightarrow 0$ can only be formed by including logarithmic terms which do not correspond to a renormalizable Lagrangian. If $d=0$ the power series solution (3.15) exists with arbitrary coefficient $\varrho_{+|\xi|}$ and represents the general solution. Thus for negative integral $\xi$ either no power series solution $\lambda_{+}$exists or $\lambda_{+}$represents the general solution of (3.5) with an arbitrary parameter.

If $\xi>0$ a power series (3.14) always exists for $\lambda_{+}$. The power series (3.13) for $\lambda_{-}$ exists provided $\xi$ is not integral. If $\xi$ is a positive integer either no power series solution (3.13) exists or it represents the general solution with arbitrary coefficient $\varrho_{-(\xi+1)}$.

For $\xi=0$ both expansions (3.13-14) coincide and exist with uniquely determined coefficients.

The case $b_{0}=0, c_{1} \neq 0$ was treated in [7]. If $\Delta>0$, there exist two distinct power series solutions $\lambda_{+}$and $\lambda_{-}$of the form (3.13-14). Although their coefficients are unique, they may include the general solution. For the difference of two solutions with the same weak coupling behavior $\varrho_{+} g^{2}$ (or $\varrho_{-} g^{2}$ respectively) is exponentially decreasing for $g \rightarrow 0$. If $b_{0}=0$ and $\Delta=0$ no power series solution of (3.5) exists unless all coefficients of terms $g^{2 n}$ in $\beta_{1}$ vanish. In the latter case $\lambda \equiv 0$ is the only power series solution.

We now discuss the simplifications which occur for supersymmetric gauge theories with $\lambda=h^{2}$, where $h$ describes a matter or Higgs interaction. In that case all coefficients of the terms $g^{2 n}$ in $\beta_{1}$ vanish. As the square of a coupling parameter $\lambda$ is non-negative. We further assume $c_{1}>0$ which is usually the case.

The absence of a $g^{4}$-term in $\beta_{1}$ implies that (3.9) is always satisfied. This eliminates a major obstacle in constructing renormalizable reduced models. The roots of (3.7) become

$$
\varrho_{0}=0 \text { and } \varrho_{0}=\frac{b_{0}-c_{2}}{c_{1}} .
$$

Since $\beta_{1}$ vanishes at $\lambda=0$, Eq. (3.5) has the solution $\lambda \equiv 0$. Apart from this trivial solution we list the following power series solutions of (3.5) under the positivity constraint $\lambda \geqq 0$ :

(1) $b_{0}<0, c_{2}<b_{0}$, or equivalently $\xi>0, \varrho_{+}>0, \varrho_{-}=0$.

There is the expansion (3.14) of $\lambda_{+}$with unique coefficients. If $\xi$ is an integer there is further an expansion of $\lambda_{-}$,

$$
\lambda_{-}=d g^{2 \xi+2}+\sum_{j=\xi+1}^{\infty} \varrho_{-j} g^{2 j+2}, \quad \xi=1,2, \ldots, d>0,
$$

with arbitrary positive coefficient $d$. 
(2a) $b_{0}>0, c_{2}<b_{0}$, or equivalently $\xi<0, \varrho_{+}>0, \varrho_{-}=0$.

If $\xi$ is not an integer there is the expansion (3.14) of $\lambda_{+}$with unique coefficients. If $\xi$ is a negative integer either $\lambda_{+}$does not exist or the coefficient $d_{+|\xi|}$ is arbitrary.

(2b) $b_{0}>0, c_{2}>b_{0}$, or equivalently $\xi<0, \varrho_{+}=0, \varrho_{-}<0$.

If $\xi$ is an integer there is the expansion

$$
\lambda_{+}=d g^{2|\xi|+2}+\sum_{j=|\xi|+1}^{\infty} \varrho_{+j} g^{2 j+2}, \quad \xi=-1,-2, \ldots, d>0 .
$$

(3) $b_{0}=0, c_{2}<0$ implying $\varrho_{+}>0, \varrho_{-}=0$.

There is the expansion (3.14) of $\lambda_{+}$with unique coefficients.

In all other cases, namely $b_{0}=c_{2}$ or $b_{0} \leqq 0$ with $c_{2} \geqq b_{0}$, there are no power series solutions except $\lambda \equiv 0$, which have $\lambda \geqq 0$ for sufficiently small $g^{2}$.

Finally we remark that the lowest order form $\lambda \approx \varrho_{0} g^{2}$ of a power series expansion can be made exact by reparametrizing $\lambda$ provided $\varrho_{0} \neq 0$. For the coefficients of

$$
\lambda^{\prime}=\lambda+a_{1} \lambda^{2}+a_{2} \lambda^{3}+\ldots
$$

can be chosen such that

$$
\lambda^{\prime} \equiv \varrho_{0} g^{2} .
$$

If $\varrho_{0}=0$ the transformation (3.19) in general does not even lead to a polynomial form of $\lambda^{\prime}$. But

$$
\lambda^{\prime}=\lambda+b_{1} \lambda g^{2}+b_{2} \lambda g^{4}+\ldots
$$

can be used to transform a power series solution

into

$$
\lambda=\varrho_{n} g^{2 n+2}+o\left(g^{2 n+4}\right)
$$

$$
\lambda^{\prime} \equiv \varrho_{n} g^{2 n+2} \text {. }
$$

\section{Model of a Spinor and Pseudoscalar Field}

We consider the massless renormalizable model of a single spinor field $\psi$ interacting with a pseudoscalar field $A$. The interaction terms are

$$
i g \bar{\psi} \gamma_{5} A \psi-\frac{\lambda}{4 !} A^{4} \text {. }
$$

The model contains two independent parameters $g$ and $\lambda$. We try to reduce the system to a renormalizable description in terms of $g$ only. In lowest order the $\beta$-functions are

$$
\begin{aligned}
& \beta_{0}=\frac{1}{16 \pi^{2}} 5 g^{4}+\ldots \\
& \beta_{1}=\frac{1}{16 \pi^{2}}\left(\frac{3}{2} \lambda^{2}+4 \lambda g^{2}-24 g^{4}\right)+\ldots
\end{aligned}
$$

From this the values

$$
\begin{gathered}
\varrho_{+}=\frac{1}{3}+\frac{1}{3} \sqrt{145}>0, \\
\varrho_{-}=\frac{1}{3}-\frac{1}{3} \sqrt{145}<0, \\
\xi=-\frac{1}{5} \sqrt{145}<0
\end{gathered}
$$


follow. If $\lambda$ is positive for sufficiently small $g$ there is only one power series solution,

$$
\lambda=\frac{1}{3}(1+\sqrt{145}) g^{2}+\varrho_{+1} g^{4}+\varrho_{+2} g^{6}+\ldots,
$$

uniquely determining $\lambda$ as a function of $g .{ }^{\star}$ By a redefinition of $\lambda$, the lowest order can be made exact

$$
\lambda=\frac{1}{3}(1+\sqrt{145}) g^{2} .
$$

For completeness we quote the generalization of (3.15) from [6]

$$
\begin{aligned}
\lambda= & \frac{1}{3}(1+\sqrt{145}) g^{2}+\varrho_{+1} g^{4}+\varrho_{+2} g^{6} \\
& +d_{11} g^{\frac{2}{5} \sqrt{145+2}}+\varrho_{+3} g^{8}+d_{12} g^{\frac{3}{5} \sqrt{145+4}} \\
& +\varrho_{+4} g^{10}+d_{21} g^{\frac{4}{5} \sqrt{145+2}}+\ldots
\end{aligned}
$$

The terms are ordered according to decreasing magnitude for $g \rightarrow 0 . \varrho_{+1}$ and $\varrho_{+2}$ are unique. $d_{11}$ is arbitrary, all other coefficients are determined for given $d_{11}$. The power series solution (4.3) is stable since the general solution (4.5) has the same asymptotic behavior for $g \rightarrow 0$. If $\lambda$ is negative for sufficiently small $g$ there is the power series

$$
\lambda=\frac{1}{3}(1-\sqrt{145}) g^{2}+\varrho_{-1} g^{4}+\varrho_{-2} g^{6}+\ldots
$$

which is an unstable solution.

\section{Wess-Zumino Model}

We study the massless Wess-Zumino model with the coupling constants $g$ and $\lambda$ of the interaction terms

$$
g \bar{\psi}\left(A+i \gamma_{5} B\right) \psi-\frac{\lambda}{2}\left(A^{2}+B^{2}\right)^{2}
$$

treated as independent parameters. In lowest order the $\beta$-functions are

$$
\begin{gathered}
\beta_{0}=\frac{1}{16 \pi^{2}} 12 g^{4}+\ldots \\
\beta_{1}=\frac{1}{16 \pi^{2}}\left(20 \lambda^{2}+8 \lambda g^{2}-16 g^{4}\right)+\ldots .
\end{gathered}
$$

From this the values

$$
\varrho_{+}=1, \quad \varrho_{-}=-\frac{4}{5}, \quad \xi=-3
$$

follow. The solutions corresponding to the supersymmetric ratio $\lambda / g^{2} \approx \varrho_{+}$have the asymptotic expansion (3.15)

$$
\lambda=g^{2}+\varrho_{+1} g^{4}+\varrho_{+2} g^{6}+\varrho_{+3} g^{8}+d g^{8} \ln g^{2}+\ldots
$$

$\varrho_{+1}, \varrho_{+2}$, and $d$ are uniquely determined. $\varrho_{+3}$ is arbitrary. The higher order coefficients are determined for given $\varrho_{+3}$. The existence of a renormalized version of the supersymmetric model implies that a power series solution of $\lambda$ exists.

* See Note added in proof on p. 225 
Therefore $d=0$, so that (5.3) takes the form

$$
\lambda=g^{2}+\sum_{j=1}^{\infty} \varrho_{+j} g^{2 j+2}
$$

with arbitrary $\varrho_{+3}$. Only one of those corresponds to the supersymmetric case. With suitable supersymmetric normalization conditions it is

$$
\lambda=g^{2}
$$

for the supersymmetric system and

$$
\lambda=g^{2}+\varrho_{+3} g^{8}+\sum_{j=4}^{\infty} \varrho_{+j} g^{2 j+2}
$$

for the asymmetric reduced systems with arbitrary $\varrho_{+3} \neq 0$. The solution (5.3) is stable since its asymptotic behavior is the same as for the general solution (5.6). In addition there is the power series solution starting with $\varrho_{-} g^{2}$,

$$
\lambda=-\frac{4}{5} g^{2}+\sum_{j=1}^{\infty} \varrho_{-j} g^{2 j+2},
$$

which is unstable and not related to supersymmetry.

Acknowledgements. I am grateful to Drs. Oehme, Piguet, and Sibold for many helpful discussions. Further I wish to thank Dr. Kuiper for a stay at the Institut des Hautes Études Scientifiques, Bures-sur-Yvette, in the spring of 1982 where part of this work was done.

\section{References}

1. Callan, C.: Broken scale invariance in scalar field theory. Phys. Rev. D 2, 1541-1547 (1970)

2. Symanzik, K.: Small distance behavior in field theory and power counting. Commun. Math. Phys. 18, 227-246 (1970)

3. Mandelstam, S.: Light-cone superspace and the ultraviolet finiteness of the $N=4$ model. Nucl. Phys. B 213, 149-168 (1983)

4. Chang, N.P.: Eigenvalue conditions and asymptotic freedom for Higgs scalar gauge theories. Phys. Rev. D 10, 2706-2709 (1974)

5. Chang, N.P., Das, A., Perez-Mercader, J.: Asymptotically free SU(5) model with three generations. Phys. Rev. D 22, 1829-1832 (1980)

6. Oehme, R., Zimmermann, W.: Relation between effective couplings for asymptotically free models. Commun. Math. Phys. (to appear)

7. Oehme, R., Sibold, K., Zimmermann, W.: Renormalization group equations with vanishing lowest order of the primary $\beta$-function (to be published in Phys. Lett.)

8. Gross, D., Wilczek, F.: Asymptotically free gauge theories. I. Phys. Rev. D 8, 3633-3652 (1973)

9. Wess, J., Zumino, B.: A Lagrangian model invariant under supergauge transformations. Phys. Lett. 49 B, 52-54 (1974)

10. Clark, T., Piguet, O., Sibold, K.: Supercurrents, renormalization and anomalies. Nucl. Phys. B 143, 445-484 (1978)

11. Maison, D.: Determination of correct $\beta$-functions for super Yang-Mills theories using a supersymmetry violating renormalization scheme. Preprint Werner-Heisenberg-Institut für Physik MPI/PTh 43/84 (to be published) 
12. Suzuki, M.: On instability of asymptotic freedom of supergauge Yang-Mills theories. Nucl. Phys. B 83, 269-275 (1974)

13. Zimmermann, W.: The renormalization group of the model of $A^{4}$-coupling in the abstract approach of quantum field theory. Commun. Math. Phys. 76, 39-64 (1980)

14. Oehme, R., Zimmermann, W.: Analyticity of effective coupling and propagators in massless models of quantum field theory. Commun. Math. Phys. 85, 363-379 (1982)

15. Symanzik, K.: On some massless superrenormalizable and non-renormalizable theories. In: Lecture Notes in Physics, Vol. 39, pp. 101-106, H. Araki (ed.). Berlin, Heidelberg, New York: Springer 1975

16. Bandelloni, G., Becchi, C., Blasi, A., Collina, R.: Renormalization of models with radiative mass generation. Commun. Math. Phys. 67, 147-178 (1978)

17. Piguet, O., Sibold, K.: Renormalizing supersymmetry without auxiliary fields. Preprint Université de Genève, UGVA-DPT 1984/10-443 (to be published)

Communicated by G. Mack

Received July 23, 1984

Note added in proof. This relation between the quartic and the Yukawa coupling constant was found independently by E. de Rafael and R. Stora (private communication). 
